\title{
Division of Cancer Treatment and Diagnosis
}

National Cancer Institute

\section{Source}

National Cancer Institute. Division of Cancer Treatment and Diagnosis. NCI Thesaurus.

Code C18751.

Improves the lives of the American public by discovering and conducting better ways to diagnose, assess, treat, and cure cancer through stimulating, coordinating, and funding a national program of cancer research. 